Pacific Journal of Mathematic 


\title{
A HAHN DECOMPOSITION FOR LINEAR MAPS
}

\author{
RICHARD I. LOEBL
}

The question is studied whether every bounded self-adjoint linear map $\varphi$ be tween two $C^{*}$-algebras can be written as the difference of bounded positive linear maps. Such a decomposition is called a Hahn decomposition of $\varphi$.

THEOREM. Let $X$ be an infinite compact Hausdorff space. Then there is a bounded, self-adjoint linear map, with domain $C(X)$, that does not admit a Hahn decomposition.

A bounded linear map $\varphi$ is said to have finite total variation if

$$
\sup \left\{\left\|\sum_{i=1}^{n}\left|\varphi\left(a_{i}\right)\right|\right\|: a_{\imath} \in \mathscr{A}, 0 \leqq a_{i}, \sum a_{i} \leqq 1\right\}<\infty .
$$

THEOREM. If the domain is commutative, and if the range is a von Neumann algebra, then a sufficient condition for a selfadjoint map to admit a Hahn decomposition is that the map have finite total variation.

o. Introduction. It is a well-known theorem [4] that every linear functional $\tau$ on a $C^{*}$-algebra $\mathscr{A}$ can be written $\tau=\tau_{1}-\tau_{2}+$ $i\left(\tau_{3}-\tau_{4}\right)$, where the $\tau_{j}$ are positive linear functionals. It is, therefore, natural to ask whether every bounded linear map $\varphi$ between two $C^{*}$-algebras $\mathscr{A}$ and $\mathscr{B}$ admits a decomposition $\varphi=\varphi_{1}-\varphi_{2}+$ $i\left(\varphi_{3}-\varphi_{4}\right)$, where the $\varphi_{j}$ are positive linear maps.

Given any bounded linear map $\varphi$, if we define the linear map $\widetilde{\varphi}$ by $\widetilde{\varphi}(a)=\varphi\left(a^{*}\right)^{*}$, it is easy to see that $\|\widetilde{\varphi}\|=\|\varphi\|$, and that $\widetilde{\varphi}$ is the natural "adjoint" map to $\varphi$. Hence, the map $\varphi_{1}=(\varphi+\widetilde{\varphi}) / 2$ is self-adjoint, i.e., $\varphi_{1}\left(a^{*}\right)=\varphi_{1}(\alpha)^{*}$, as is $\varphi_{2}=(\varphi-\widetilde{\varphi}) / 2 i$, and therefore $\varphi$ can be written (uniquely) as $\varphi=\varphi_{1}+i \varphi_{2}$, the usual combination of self-adjoint elements.

We are now reduced to the following problem: Given a bounded, self-adjoint linear map $\varphi$ between two $C^{*}$-algebras, when can we write $\varphi=\varphi_{1}-\varphi_{2}$ where $\varphi_{1}, \varphi_{2}$ are bounded, positive linear maps?

Definition 0.1. We shall call such a form a Hahn decomposition of $\varphi$.

In general, a Hahn decomposition is not always possible. Even in the commutative case, pathology can occur [see Theorem 2.2 below].

For future references, we state here Grothendieck's result for 
functionals on a $C^{*}$-algebra [4].

THEOREM 0.2. Let $\varphi$ be a bounded self-adjoint functional on a $C^{*}$-algebra. Then we can write $\varphi=\varphi^{+}-\varphi^{-}$, with $\varphi^{+}, \varphi^{-}$positive, and $\|\varphi\|=\left\|\varphi^{+}\right\|+\left\|\varphi^{-}\right\|$.

In our terminology, bounded self-adjoint functionals admit a Hahn decomposition.

Most of this material appeared in my Ph. D. thesis, Berkeley, 1973, written under the direction of Professor William B. Arveson. During the course of this research, I benefitted greatly from conversations with Professors Arveson, Oscar Lanford, and Donald Sarason.

1. Preliminaries. In this section, we shall study some simple cases where a Hahn decomposition exists, and some consequences of the decomposition. As usual, $M_{n}$ denotes the $C^{*}$-algebra of $n \times n$ complex matrices.

Lemma 1.1. Let $\mathscr{A}$ be a $C^{*}$-algebra, let $\varphi: \mathscr{A} \rightarrow M_{n}$ be a bounded linear self-adjoint map. Then we can write $\varphi(x)=$ $\sum_{j=1}^{N} \rho_{j}(x) A_{j}$, where the $\rho_{j}$ are bounded self-adjoint functionals, $\left\|\rho_{j}\right\| \leqq\|\varphi\|$, and $A_{j} \in M_{n}$ satisfy $A_{j}=A_{j}^{*},\left\|A_{j}\right\| \leqq 1$, and $A_{j}^{2}$ is a projection.

Proof. Let $\left\{E_{j_{k}}\right\}$ denote the usual basis of $M_{n}$, let $z_{1}, \cdots, z_{n}$ be the canonical basis for $\boldsymbol{C}^{n}$. Define the functionals $\varphi_{j k}$ by $\varphi_{j k}(x)=$ $\left(\varphi(x) z_{k}, z_{j}\right)$. Then $\left\|\varphi_{j k}\right\| \leqq\|\varphi\|$, and we have $\varphi=\sum \varphi_{j_{k}} E_{j_{k}}$. Since $\phi$ is self-adjoint, it is easy to see that the functionals $\phi_{j_{k}}$ satisfy $\varphi_{j_{k}}=\widetilde{\varphi}_{k j}$. In particular the $\varphi_{j j}$ are self-adjoint. Hence

$$
\begin{aligned}
\varphi= & \sum_{j=1}^{n} \varphi_{j j} E_{j j}+\sum_{j \neq k} \varphi_{j k} E_{j k}=\sum \varphi_{j j} E_{j j}+\sum_{j \neq k}\left(\frac{\varphi_{j k}+\varphi_{k j}}{2}\right)\left(E_{j k}+E_{k j}\right) \\
& +\sum_{j \neq k}\left(\frac{\varphi_{j k}-\varphi_{k j}}{2 i}\right)\left(\frac{E_{k j}-E_{j k}}{i}\right)
\end{aligned}
$$

which writes $\varphi$ in the promised manner.

Proposition 1.2. Let $\mathscr{A}$ be a $C^{*}$-algebra, let $\varphi$ : $\mathscr{A} \rightarrow M_{n}$ be a bounded, linear, self-adjoint map. Then $\varphi$ admits a Hahn decomposition.

Proof. Write $\varphi=\sum \rho_{j} A_{j}$ as in 1.1. Then we can apply Theorem 0.2 to say $\rho_{j}=\rho_{j}^{+}-\rho_{j}^{-}$and $\left\|\rho_{j}\right\|=\left\|\rho_{j}^{+}\right\|+\left\|\rho_{j}^{-}\right\|$. We 
also write $A_{j}=A_{j}^{+}-A_{j}^{-}$, with $\left\|A_{j}\right\|=\max \left(\left\|A_{j}^{+}\right\|,\left\|A_{j}^{-}\right\|\right)$, where the $A_{j}^{+}, A_{j}^{-}$are positive. Then $\rho_{j} A_{j}=\left(\rho_{j}^{+} A_{j}^{+}+\rho_{j}^{-} A_{j}^{-}\right)-\left(\rho_{j}^{+} A_{j}^{-}+\rho_{j}^{-} A_{j}^{+}\right)$, is the difference of positive maps, and hence $\varphi=\sum \rho_{j} A_{j}$ is the difference of positive maps.

\section{REMARKS 1.3.}

(i) We do not get a good bound on $\left\|\varphi^{+}\right\|,\left\|\varphi^{-}\right\|$from this proof. However, we will see later [see Remark 2.3(ii)] that good bounds cannot be obtained without more detailed knowledge of $\varphi$.

(ii) We say that a linear map $\varphi$ between two Banach spaces $X$ and $Y$ is nuclear if we can write $\varphi=\sum \rho_{i} \otimes y_{i}$, where $\rho_{i} \in X^{\prime}$ and $y_{i} \in Y$, with $\sum\left\|\rho_{i}\right\|\left\|y_{i}\right\|<\infty$, and $\left(\rho_{i} \otimes y_{j}\right)(x)=\rho_{i}(x) y_{i}$. Let \|\|$\varphi \mid \|=\inf \left\{\sum\left\|\rho_{i}\right\|\left\|y_{i}\right\|: \varphi=\sum \rho_{i} \otimes y_{i}\right\}$, then if $\|\varphi\| \mid<\infty$, where $\varphi$ is a bounded linear map of $C^{*}$-algebras, the proof of Proposition 1.2 shows that $\varphi$ admits a Hahn decomposition. Unfortunately, most linear maps are not nuclear.

(iii) For the case of a bounded self-adjoint linear map from $C(X) \rightarrow M_{n}$, we are able to sketch another proof of the Hahn decomposition which is very tempting to generalize to $\mathscr{L}(H)$. Note that using Lemma 1.5, we see that every bounded linear map of $C(X) \rightarrow M_{n}$ is completely bounded [Definition 1.4].

Sketch of proof. Let $\varphi: C(X) \rightarrow M_{n}$ be bounded, linear, selfadjoint. Then $\varphi_{i j}(f)=\left(\varphi(f) z_{j}, z_{i}\right)$ is a bounded linear functional on $C(X)$, hence there is a bounded Borel measure $\mu_{i j}$ such that $\varphi_{i j}(f)=$ $\int f d \mu_{i j}$. Since $\varphi$ is self-adjoint, we have $\mu_{i i}$ is real, and $\mu_{j i}=\overline{\mu_{i j}}$. Let $\mu=1 / n^{2} \sum_{i, j=1}^{n}\left|\mu_{i j}\right|$, then $\mu$ is positive, $\|\mu\| \leqq\|\varphi\|$, and each $\mu_{i j}$ is absolutely continuous with respect to $\mu$. Hence, there exist functions $h_{\imath j} \in L^{1}(\mu)$, such that $d \mu_{\imath j}=h_{i j} d \mu$. Let $H(x)=\left(h_{i j}(x)\right)$, then $H(x)$ is self-adjoint a.e. $(d \mu)$. By the finite-dimensional spectral theorem, $H(x)=P(x)-Q(x)$, where $P(x), Q(x)$ are positive a.e. $(d \mu)$. Then $\varphi(x)=\int f(x) H(x) d \mu=\int f(x) P(x) d \mu-\int f(x) Q(x) d \mu$ is the difference of positive maps. In fact, $K(x)=\|H(x)\| \in L^{1}(\mu)$.

Definitions 1.4. Let $\varphi: \mathscr{A} \rightarrow \mathscr{B}$ be a linear map of $C^{*}$-algebras. Let $M_{n}$ be the $n \times n$ matrices, and let $\varphi_{n}$ be the natural map from $\mathscr{A} \otimes M_{n} \rightarrow \mathscr{B} \otimes M_{n}$. Then $\varphi$ is completely positive if all $\varphi_{n}$ are positive ([7]), and completely bounded if $\sup _{n}\left\|\varphi_{n}\right\|<\infty$ ([1]).

A completely positive map is completely bounded ([1]), and if either $\mathscr{A}$ or $\mathscr{B}$ is commutative, a positive map is completely positive $([1,7])$.

LEMMA 1.5. Let $\varphi: \mathscr{A} \rightarrow \mathscr{B}$ be a bounded self-adjoint map of 
$C^{*}$-algebras. In case either $\mathscr{A}$ or $\mathscr{B}$ is abelian, a necessary condition for $\varnothing$ to admit a Hahn decomposition is that $\varphi$ be completely bounded.

Proof. Suppose $\varphi=\varphi^{+}-\varphi^{-}$is a Hahn decomposition. Then both $\varphi^{+}$and $\varphi^{-}$are completely positive, hence completely bounded. Hence, $\left\|\varphi_{N}\right\|=\left\|\varphi_{N}^{+}-\varphi_{N}^{-}\right\| \leqq\left\|\varphi_{N}^{+}\right\|+\left\|\varphi_{N}^{-}\right\| \leqq M_{1}+M_{2}, \forall N$, so $\varphi$ is completely bounded.

REMARK. It is not essential in Lemma 1.5 that $\varphi$ be self-adjoint.

2. A counterexample. We now proceed with a modification of an example due to O. E. Lanford, showing that a Hahn decomposition is not always possible.

LeMma 2.1. Let $n \geqq 1$. Then there exist $A_{1}, \cdots, A_{n} \in M_{2^{n}}$ such that

(1) $A_{i}=A_{i}^{*}$

(2) $A_{i} A_{j}+A_{j} A_{i}=2 \delta_{i j} \cdot I_{2^{n}}$

(3) $\operatorname{Tr}\left(A_{i}\right)=0$.

Proof. Let $H=\left(\begin{array}{ll}0 & 1 \\ 1 & 0\end{array}\right), \quad J=\left(\begin{array}{rr}1 & 0 \\ 0 & -1\end{array}\right), \quad I=I_{2}=\left(\begin{array}{ll}1 & 0 \\ 0 & 1\end{array}\right) . \quad$ Then $H^{*}=H, I^{*}=I, J^{*}=J, H^{2}=I, J^{2}=I$, and $H J+J H=0$. Now, let

$$
\begin{aligned}
& A_{1}=H \otimes \overbrace{I \otimes \cdots \otimes I}^{n-1} \\
& A_{2}=J \otimes H \otimes \overbrace{I \otimes \cdots \otimes I}^{n-2} \\
& \vdots \\
& A_{k}=\overbrace{J \otimes \cdots \otimes}^{k-1} J \otimes H \otimes \overbrace{I \otimes \cdots \otimes I}^{n-k} \text { for } 3 \leqq k \leqq n .
\end{aligned}
$$

Clearly $A_{i}=A_{2}^{*}, A_{i}^{2}=I$, and $\operatorname{Tr}\left(A_{i}\right)=0$. For $j>1, A_{1} A_{j}+A_{j} A_{1}=0$ by looking at the first factor. If $1<i<j \leqq n$, then the $i^{\text {th }}$ factor of $A_{i}$ is $H$, and the $i^{\text {th }}$ factor of $A_{j}$ is $J$, so we have $A_{i} A_{j}+A_{j} A_{i}=0$.

We remark that the $A_{i}$ generate a Clifford algebra [6].

Note that if $A \in M_{2^{n}}$, and $A=\sum \alpha_{i} A_{i}$, where $A_{i}$ are from Lemma 2.1 , then $A^{*} A+A A^{*}=\sum \bar{\alpha}_{j} \alpha_{i} A_{j} A_{i}+\sum \alpha_{i} \bar{\alpha}_{j} A_{i} A_{j}=\sum \alpha_{i} \alpha_{j}\left[A_{i} A_{j}+\right.$ $\left.A_{j} A_{i}\right]=\sum \alpha_{i} \bar{\alpha}_{j}\left(2 \delta_{i j} I\right)=2\left(\sum\left|\alpha_{i}\right|^{2}\right) I$. Thus $\|A\| \leqq \sqrt{2} \sqrt{\sum\left|\alpha_{i}\right|^{2}}$; that is, the $A_{i}$ are almost an orthonormal basis for their span.

Now, let $X$ be an infinite compact Hausdorff space, and let $\rho_{1}, \cdots, \rho_{n}$ be positive linear functionals on $C(X)$ such that the $\rho_{i}$ have disjoint closed supports and $\left\|\rho_{i}\right\|=n^{-3 / 4}$. Let $\varphi^{(n)}: C(X) \rightarrow M_{2^{n}}$ by $\quad \varphi^{(n)}(f)=\sum_{i=1}^{n} \rho_{i}(f) A_{i}$. Then $\left\|\varphi^{(n)}(f)\right\| \leqq \sqrt{2} \sqrt{\sum\left|\rho_{i}(f)\right|^{2}} \leqq$ 
$\sqrt{2} \sqrt{\sum\left(n^{-3 / 4}\|f\|\right)^{2}}=\sqrt{2}\|f\| \sqrt{\sum n^{-3 / 2}}=\sqrt{2}\|f\| n^{-1 / 4} \leqq \sqrt{2}\|f\|$. Note also that $\varphi^{(n)}$ is a self-adjoint linear map.

THEOREM 2.2. Let $X$ be a compact Hausdorff space such that card $(X)=\infty$. Then there is a bounded, self-adjoint linear map $\varphi$ from $C(X)$ into the compact operators on a separable Hilbert space such that $\varphi$ does not admit a Hahn decomposition.

Proof. Since card $(X)=\infty$, for every integer $n \geqq 1$, we can find positive linear functionals $\rho_{1}^{(n)}, \cdots, \rho_{n}^{(n)}$ such that $\left\|\rho_{i}^{(n)}\right\|=n^{-3 / 4}$, and all the $\rho_{i}^{(n)}$ have disjoint closed supports, for $1 \leqq i \leqq n, 1 \leqq n$. Let $\mathscr{C}_{n}=C^{2^{n}}$, and let $\mathscr{H}=\bigoplus_{n} \mathscr{H}_{n}$. Let $\varphi: C(X) \rightarrow \mathscr{L}(H)$ by $\varphi(f)=\bigoplus \varphi^{(n)}(f)$, where $\varphi^{(n)}(f)$ is as above.

Then $\|\varphi\|=\sup _{n}\left\|\varphi^{(n)}\right\| \leqq \sqrt{2}$. Let $\widetilde{\varphi}^{(N)}=\bigoplus_{n=1}^{N} \varphi^{(n)}$, then $\widetilde{\varphi}^{(N)}(f)$ has finite rank for all $f \in C(X)$, and

$$
\left\|\varphi-\widetilde{\Phi}^{(N)}\right\|=\sup _{n>N}\left\|\varphi^{(n)}\right\| \leqq \sup _{n>N} \sqrt{2} n^{-1 / 4}=\sqrt{2}(N+1)^{-1 / 4} \rightarrow 0
$$

as $N \rightarrow \infty$. Hence $\varphi(f)$ is compact for all $f \in C(X)$.

We shall give two proofs that $\varphi$ does not admit a Hahn decomposition. In the first proof, we shall show $\varphi$ is not completely bounded, so that Lemma 1.5 implies $\varphi$ does not admit a Hahn decomposition.

Let $\varphi_{k}=\varphi \otimes \mathrm{id}_{k}: C(X) \otimes M_{k} \rightarrow \mathscr{L}(H) \otimes M_{k}$; then it is easy to see that $\varphi_{k}=\bigoplus_{n}\left(\varphi^{(n)} \otimes \mathrm{id}_{k}\right)$, so $\left\|\varphi_{k}\right\|=\sup _{n}\left\|\varphi_{k}^{(n)}\right\|$ : We will show that $\left\|\varphi_{2^{n}}^{(n)}\right\| \geqq n^{1 / 4}$, hence $\left\|\varphi_{2^{n}}\right\| \geqq n^{1 / 4}$, and thus $\varphi$ is not completely bounded.

For convenience of notation, let $\psi=\varphi^{(n)}=\sum \rho_{i} A_{i}$. In $C(X) \otimes$ $M_{2^{n}}=C\left(X, M_{2^{n}}\right)$, consider the matrix $F(x)=\left(f_{i j}(x)\right)$ such that on the support $K_{i}$ of $\rho_{i}, F(x) \equiv A_{i}$, and such that otherwise $F(x)$ is a convex combination of the $A_{i}$. Such an $F$ can be constructed in the following manner: Since the supports $K_{i}$ of the $\rho_{i}$ are disjoint, a slight variation of the usual partition of unity argument yields continuous functions $g_{1}, \cdots, g_{n}$ such that $0 \leqq g_{i} \leqq 1, \sum g_{i} \equiv 1, g_{i} \equiv 1$ on $K_{i}$; we then let $F(x)=\sum g_{i}(x) A_{i}$. Note that $F(x)=\overline{F(x)} \forall x$, and $\|F\|=\sup _{x}\|F(x)\|=1$.

We have that $\psi_{2^{n}}(F)=\left(\psi \otimes \mathrm{id}_{2^{n}}\right)(F)=\left(\sum\left(\rho_{i} \otimes A_{i}\right) \otimes \mathrm{id}_{2^{n}}\right)(F)=$ $\sum A_{i} \otimes\left(\rho_{i} \otimes \mathrm{id}_{2^{n}}(F)\right)=\sum A_{i} \otimes\left\|\rho_{\imath}\right\| A_{i}=\sum\left\|\rho_{i}\right\| A_{i} \otimes A_{i}$. Since $A_{i} A_{j}+$ $A_{j} A_{i}=0$ for $i \neq j$, we see that $A_{i} A_{j}=-A_{j} A_{i}$ for $i \neq j$, and hence $A_{i} \otimes A_{i}$ commutes with $A_{j} \otimes A_{j}$ ! It is clear that $\left\|\psi_{2^{n}}(F)\right\| \leqq$ $\sum\left\|\rho_{\imath}\right\|\left\|A_{i} \otimes A_{i}\right\|=\sum\left\|\rho_{i}\right\|$. We claim: there is a unit vector $z \epsilon$ $C^{2^{n}} \otimes C^{2^{n}} \quad$ such that $\left(A_{i} \otimes A_{i}\right)(z)=z, \quad \forall i . \quad$ If $\quad$ so, $\quad\left\|\psi_{2}^{n}(F)\right\| \geqq$ $\left|\left\langle\psi_{2^{n}}(F)(z), z\right\rangle\right|=\sum\left\|\rho_{i}\right\|$, so we actually have $\left\|\psi_{2^{n}}(F)\right\|=\sum\left\|\rho_{i}\right\|$. Thus $\left\|\psi_{2^{n}}\right\| \geqq\left\|\psi_{2^{n}}(F)\right\|=\sum\left\|\rho_{i}\right\|=\sum_{l=1}^{n} n^{-3 / 4}=n^{1 / 4}$, as desired. (We 
showed that $\left\|\psi_{2^{n}}\right\| \geqq \sum\left\|\rho_{i}(F)\right\|$ in order that the role of the actual values of $\left\|\rho_{i}\right\|$ can be seen. In fact, we have really shown that $\left.\left\|\psi_{2^{n}}\right\|=\sum\left\|\rho_{i}\right\|.\right)$

Now, to prove the claim, we need some observations.

(1) There is a unitary operator $U \in M_{2^{n}}$ so that $U^{*} A_{i} U=-A_{i}$

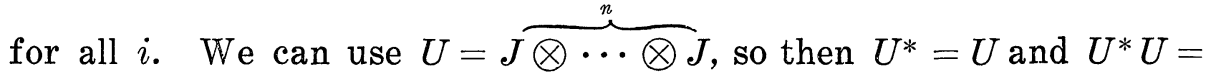
$U U^{*}=\overbrace{J^{2} \otimes \cdots \otimes}^{n} J^{2}=\overbrace{I \otimes \cdots \otimes I}^{n}=I$. We then have

$$
\begin{aligned}
& U * A_{i} U=[J \otimes \cdots \otimes J][\overbrace{J \otimes \cdots \otimes J}^{i-1} \otimes H \otimes \overbrace{I \otimes \cdots \otimes I}^{n-i}][J \otimes \cdots \otimes J] \\
& =\overbrace{J \otimes \cdots \otimes}^{i-1} J \otimes J H J \otimes \overbrace{I \otimes \cdots \otimes I}^{n-i},
\end{aligned}
$$

but $H J+J H=0$ means $J H J=-H$, hence $U^{*} A_{1} U=-A_{i}$.

(2) Let $i_{1}, \cdots, i_{k}$ be distinct; then $\operatorname{Tr}\left(A_{i_{1}} \cdot A_{i_{2}} \cdots A_{i_{k}}\right)=0$. For if $k$ is odd,

$$
\begin{aligned}
\operatorname{Tr}\left(A_{i_{1}} A_{i_{2}} \cdots A_{i_{k}}\right) & =\operatorname{Tr}\left(U^{*} A_{i_{1}} A_{i_{2}} \cdots A_{i_{k}} U\right) \\
& =\operatorname{Tr}_{\mathrm{g}}^{\mathrm{g}}\left(U^{*} A_{i_{1}} U \cdot U^{*} A_{i_{2}} U \cdots U^{*} A_{i_{k}} U\right) \\
& =\operatorname{Tr}\left(-A_{i_{1}} \cdot A_{\imath_{2}} \cdots-A_{i_{k}}\right) \\
& =(-1)^{k} \operatorname{Tr}\left(A_{i_{1}} \cdots A_{i_{k}}\right),
\end{aligned}
$$

so $\operatorname{Tr}\left(A_{i_{1}} A_{i_{2}} \cdots A_{i_{k}}\right)=0$.

If $k$ is even, then

$\operatorname{Tr}\left(A_{i_{1}} A_{i_{2}} \cdots A_{i_{k}}\right)=\operatorname{Tr}\left(A_{i_{k}} A_{i_{1}} A_{\imath_{2}} \cdots A_{i_{k-1}}\right)=(-1)^{k-1} \operatorname{Tr}\left(A_{i_{1}} A_{i_{2}} \cdots A_{\imath_{k}}\right)$ by 2.1, so again $\operatorname{Tr}\left(A_{i_{1}} A_{i_{2}} \cdots A_{i_{k}}\right)=0$.

(3) Let $S_{i}=A_{i} \otimes A_{i}, 1 \leqq i \leqq n$. Then the $S_{i}$ are mutually commuting, self-adjoint, and have square $=I$. This follows easily from Lemma 2.1. Further, the $S_{i}$ can be simultaneously diagonalized, and it follows that the eigenvalues of the $S_{i}$ are all +1 or -1 . Also, $S_{i}=2 P_{i}-I$, where the $P_{i}$ are commuting projections.

Now, to show that the $S_{i}$ do indeed have a common +1 eigenvalue, it suffices to consider the $P_{i}$, for

$$
\operatorname{dim}\left(\Pi P_{i}\right)=\operatorname{dim}\left(\text { common }+1 \text { eigenspace of the } S_{i}\right) \text {. }
$$

But

$$
\begin{aligned}
\operatorname{dim}\left(\Pi P_{i}\right) & =\operatorname{Tr}\left(\Pi P_{i}\right)=\operatorname{Tr}\left[\left(\frac{I+S_{1}}{2}\right) \cdots\left(\frac{I+S_{n}}{2}\right)\right] \\
& =2^{-n} \operatorname{Tr}\left[\left(I+S_{1}\right) \cdots\left(I+S_{n}\right)\right] \\
& =2^{-n} \operatorname{Tr}\left[I+\text { products of the } S_{i}\right], \text { which by observation (2) is, } \\
& =2^{-n}[\operatorname{Tr}(I)+0 \cdots+0]=2^{-n} 2^{2 n}=2^{n} .
\end{aligned}
$$


Thus, the $S_{i}$ do indeed have many common +1 eigenvalues, proving the claim, and completing the argument.

I am indebted to W. Arveson and O. Lanford for simplifications of my original argument.

The following proof of the impossibility of a Hahn decomposition is essentially Lanford's original proof:

Proof. Suppose $\theta, \tau$ are bounded positive linear maps such that $\varphi=\theta-\tau$. Then $\theta \geqq \varphi$, and hence the compression $\theta_{n}=\left.P_{\mathscr{K}_{n}} \theta\right|_{\mathscr{C}_{n}}$ of $\theta$ to each $\mathscr{H}_{n}$ satisfies $\theta_{n} \geqq \varphi^{(n)}$. Also, $\left\|\theta_{n}\right\| \leqq\|\theta\|$, since $\theta_{n}$ is a compression of $\theta$.

From Lemma 2.1, we know that $\operatorname{Tr}\left(A_{i}\right)=0$. Let $g_{1}, \cdots, g_{n}$ be the functions we previously constructed. Then $\theta_{n}\left(g_{i}\right) \geqq \varphi^{(n)}\left(g_{i}\right)=$ $\left\|\rho_{i}\right\| A_{i}$. Choose a basis for $C^{2^{n}}$ with respect to $A_{i}$. Then the matrix representation of $\theta_{n}\left(g_{i}\right)$ is as a positive matrix, so all its diagonal elements are nonnegative. But $\theta_{n}\left(g_{1}\right) \geqq\left\|\rho_{i}\right\| A_{i}$, so $\operatorname{Tr}\left(\theta_{n}\left(g_{i}\right)\right) \geqq 2^{n-1}\left\|\rho_{i}\right\|+2^{n-1} \cdot 0$, where $\left\|\rho_{i}\right\|$ terms arise from +1 eigenvalues of $A_{i}$, 0 's from -1 eigenvalues of $A_{i}$ (since diagonal elements of $\theta_{n}\left(g_{i}\right)$ are nonnegative).

Hence $\theta_{n}(1)=\sum_{i} \theta_{n}\left(g_{i}\right)$, so $\operatorname{Tr}\left(\theta_{n}(1)\right)=\sum \operatorname{Tr}\left(\theta_{n}\left(g_{i}\right)\right) \geqq 2^{n-1} \sum\left\|\rho_{i}\right\|$. Thus $\quad\left\|\theta_{n}(1)\right\| \geqq 1 / 2^{n} \operatorname{Tr}\left(\theta_{n}(1)\right) \geqq 1 / 2 \sum_{1}^{n}\left\|\rho_{i}\right\|=1 / 2 \sum n^{-3 / 4}=(1 / 2) n^{1 / 4}$. But $\theta_{n}$ is positive, so $\left\|\theta_{n}\right\|=\left\|\theta_{n}(1)\right\|$ (see [1]) and so $(1 / 2) n^{1 / 4} \leqq$ $\left\|\theta_{n}\right\| \leqq\|\theta\|$. This is true for all $n$, hence $\theta$ is unbounded, so $\varphi$ does not admit a Hahn decomposition.

REMARKS 2.3.

(i) Basically, all that was needed was that the $\rho_{\imath}$ had disjoint supports, that $\sum_{i}^{n}\left\|\rho_{i}\right\|^{2} \leqq k$ (independent of $n$ ), and that $\sum_{1}^{n}\left\|\rho_{i}\right\| \rightarrow \infty$ as $n \rightarrow \infty$. It is interesting to note that this same quantity, $\sum\left\|\rho_{i}\right\|$, appears in both arguments.

(ii) We have shown that if $P \geqq 0, P \geqq \varphi^{(n)}$, then $\|P\| \geqq(1 / 2) n^{1 / 4}$. Hence, although $\left\|\varphi^{(n)}\right\| \leqq 2 n^{-1 / 4}$, we see that the positive part of $\varphi^{(n)}$ (from Proposition 1.2) has norm $\geqq(1 / 2) n^{1 / 4}$.

The mappings $\varphi^{(n)}$ we have used in this example, have some other interesting properties. Again, for convenience, we let $\psi=\phi^{(n)}$, so $\psi=\sum_{1}^{n} \rho_{i} A_{i}$.

Let $\left\{f_{j}\right\}, 1 \leqq j \leqq J$, be in $C(X)$ such that $\sum\left|f_{j}(x)\right|^{2} \leqq 1$ for all $x \in X$. Let $G \in C(X) \otimes M_{J}$ be

$$
G=\left(\begin{array}{cc}
f_{1} & \\
\vdots & 0 \\
f_{J} &
\end{array}\right) .
$$


Then $\left\|G^{*} G\right\|=\|G\|^{2}=\left\|\sum\left|f_{j}(x)\right|^{2}\right\| \leqq 1$. (For ease of notation, we have put the $\left\{f_{j}\right\}$ into the first column, but the following argument is valid as long as the $\left\{f_{j}\right\}$ all lie in the same row or column.)

Consider $\left(\psi \otimes \mathrm{id}_{J}\right)(G)$. Then $\left\|\left(\psi \otimes \mathrm{id}_{J}\right)(G)\right\|^{2}=\|\left(\psi \otimes \mathrm{id}_{J}(G)\right)^{*}$ $\left(\psi \otimes \mathrm{id}_{j}(G)\right)\|=\| s I+\sum_{1 \leqq j<k \leqq n} i Z_{j k} t_{j k} \|$ where (1) $s=\sum_{j, k}\left|\rho_{k}\left(f_{j}\right)\right|^{2}$; (2) $i Z_{j k}=A_{j} A_{k}$, so $Z_{j k}=Z_{j k}^{*}, Z_{j k}^{2}=I$ and $\left(2^{*}\right) Z_{j k} Z_{l m}+Z_{l m} Z_{j k}=0$ if one common index, $Z_{j_{k}} Z_{l m}=Z_{l m} Z_{j_{k}}$ if no common index; (3) $t_{j_{k}}=$ $\sum_{i}\left[\overline{\rho_{j}\left(f_{i}\right)} \rho_{k}\left(f_{i}\right)-\rho_{j}\left(f_{i}\right) \overline{\rho_{k}\left(f_{i}\right)}\right]$.

We claim: (a) $s \leqq \sum\left\|\rho_{k}\right\|^{2}$; (b) $\left|t_{j k}\right| \leqq 2\left\|\rho_{j}\right\|\left\|\rho_{k}\right\|$.

If so, then $\left\|s I+\sum_{1 \leqq j<k} i Z_{j_{k}} t_{j k}\right\| \leqq s+\left\|\sum_{k=2}^{n} i Z_{1 k} t_{1 k}\right\|+$ $\sum_{k=3}^{n} i Z_{2 k} t_{2 k}\|+\cdots+\| i Z_{n-1, n} t_{n-1, n} \|$; but the use of $\left(2^{*}\right)$ shows that, e.g.,

$$
\left\|\sum_{k=2}^{n} i Z_{1 k} t_{1 k}\right\| \leqq 2 \sqrt{\sum_{k=2}^{n}\left|t_{1 k}\right|^{2}}
$$

so we obtain

$$
\begin{aligned}
\| s I & +\sum_{1 \leqq j<k} i Z_{j k} t_{j k}\left\|\leqq \sum\right\| \rho_{k} \|^{2}+2 \sqrt{\sum_{k=2}^{n}\left|t_{1 k}\right|^{2}} \\
& +2 \sqrt{\sum_{k=3}^{n}\left|t_{2 k}\right|^{2}}+\cdots+2 \sqrt{\left|t_{n-1, n}\right|^{2}} .
\end{aligned}
$$

We now notice that $\left\|\rho_{k}\right\|=1 / n^{1-\varepsilon}, 0<\varepsilon<1 / 2$, is essentially the weakest estimate needed for $\varphi^{(n)}$ to have the desired properties (see Remark 2.3i).

Using Claim (a), we get $s \leqq n\left(1 / n^{2-2 \varepsilon}\right)=n^{2 \varepsilon} / n$, and by (b), $\left|t_{j k}\right| \leqq 2\left(n^{2 \varepsilon} / n^{2}\right)$. Hence

$$
\begin{aligned}
& \left\|s I+\sum_{1 \leqq j<k}+i Z_{j k} t_{j n}\right\| \\
& \leqq \frac{n^{2 \varepsilon}}{n}+2 \sqrt{\sum_{k=2}^{n}\left(\frac{2 n^{2 \varepsilon}}{n^{2}}\right)^{2}}+2 \sqrt{\sum_{k=3}^{n}\left(\frac{2 n^{2 \varepsilon}}{n^{2}}\right)^{2}}+\cdots+2 \sqrt{\left(\frac{2 n^{2 \varepsilon}}{n^{2}}\right)^{2}} \\
& =\frac{n^{2 \varepsilon}}{n}+4 \frac{n^{2 \varepsilon}}{n^{2}}(\sqrt{n-1}+\cdots+\sqrt{1}) \\
& \leqq \frac{n^{2 \varepsilon}}{n}+4 \frac{n^{2 \varepsilon}}{n^{2}}\left(n^{3 / 2}\right) \leqq 5 \text { for } 0<\varepsilon \leqq 1 / 4 .
\end{aligned}
$$

This means that we can choose the maps $\varphi^{(n)}$ so that $\varphi=\bigoplus \varphi^{(n)}$ has range in the compact operators, and such that $\varphi$ is not completely bounded, hence does not admit a Hahn decomposition, but such that $\varphi$ is completely row or column bounded, in the sense of the above calculation.

We now return to the proofs of Claims (a) and (b).

Proof of Claim (a). $\quad s=\sum_{i, k}\left|\rho_{k}\left(f_{i}\right)\right|^{2}=\sum_{k}\left(\sum_{i}\left|\rho_{k}\left(f_{i}\right)\right|^{2}\right)$, which 
by the generalized Schwarz inequality [3], is

$$
\leqq \sum_{k}\left(\sum_{i}\left\|\rho_{k}\right\| \rho_{k}\left(\left|f_{i}\right|^{2}\right)\right)=\sum_{k}\left\|\rho_{k}\right\|\left(\sum_{i} \rho_{k}\left(\left|f_{i}\right|^{2}\right)\right) \leqq \sum_{k}\left\|\rho_{k}\right\| \rho_{k}(1),
$$

since $\sum\left|f_{i}\right|^{2} \leqq 1$, but $\left\|\rho_{k}\right\|=\rho_{k}(1)$.

Proof of Claim (b). $\left|t_{j_{k}}\right| \leqq 2 \sum_{i}\left|\rho_{j}\left(f_{i}\right)\right|\left|\rho_{k}\left(f_{i}\right)\right|$; by the Schwarz inequality, $\quad\left|\rho_{j}\left(f_{i}\right)\right| \leqq \sqrt{\left\|\rho_{j}\right\|} \sqrt{\rho_{j}\left(\left|f_{i}\right|^{2}\right) ;} \quad$ similarly, $\quad\left|\rho_{k}\left(f_{i}\right)\right| \leqq$ $\sqrt{\left\|\rho_{k}\right\|} \sqrt{\rho_{k}\left(\left|f_{i}\right|^{2}\right)}$. Hence,

$$
\begin{aligned}
\left|t_{j k}\right| & \leqq 2 \sum_{i} \sqrt{\left\|\rho_{j}\right\|\left\|\rho_{k}\right\|} \sqrt{\rho_{j}\left(\left|f_{i}\right|^{2}\right) \rho_{k}\left(\left|f_{i}\right|^{2}\right)} \\
& =2 \sqrt{\left\|\rho_{j}\right\|\left\|\rho_{k}\right\|} \sum_{i} \sqrt{\rho_{j}\left(\left|f_{i}\right|^{2}\right)} \sqrt{\rho_{k}\left(\left|f_{i}\right|\right)^{2}} .
\end{aligned}
$$

Let

$$
x_{i}=\sqrt{\sigma_{j}\left(\left|f_{i}\right|^{2}\right)}, \quad y_{i}=\sqrt{\rho_{k}\left(\left|f_{i}\right|^{2}\right)},
$$

then the usual Schwarz inequality shows $\sum_{i} x_{i} y_{i} \leqq\left(\sum x_{i}^{2}\right)^{1 / 2}\left(\sum y_{i}^{2}\right)^{1 / 2}=$ $\left(\sum_{i} \rho_{j}\left(\left|f_{i}\right|^{2}\right)\right)^{1 / 2}\left(\sum_{i} \rho_{k}\left(\left|f_{i}\right|^{2}\right)\right)^{1 / 2} \leqq\left(\rho_{j}(1)\right)^{1 / 2}\left(\rho_{k}(1)\right)^{1 / 2}=\left\|\rho_{j}\right\|^{1 / 2}\left\|\rho_{k}\right\|^{1 / 2}$.

3. Finite total variation. As we have seen, self-adjoint linear mappings of $C^{*}$-algebras do not necessarily admit a Hahn decomposition, even in extremely nice cases. However, for the case of selfadjoint linear mappings $\varphi$ from $C(X)$ into a $W^{*}$-algebra, we have been able to obtain a sufficient condition for $\varphi$ to admit a Hahn decomposition.

Definition 3.1. Let $\varphi: \mathscr{A} \rightarrow \mathscr{B}$ be a bounded linear mapping of $C^{*}$-algebras. We say that $\varphi$ has finite total variation if

$$
V(\varphi)=\sup \left\{\left\|\sum_{k=1}^{n}\left|\varphi\left(f_{k}\right) \|_{\|}\right|: 0 \leqq f_{k} \in \mathscr{A}, \sum f_{k} \leqq 1\right\}<\infty .\right.
$$

When $\mathscr{A}=C(X)$, and $\mathscr{B}=C$, then $\varphi(f)=\int f d \mu$, for some bounded Borel measure $\mu$, and in that case $V(\varphi)$ is nothing but the total variation of the measure $\mu$.

A positive map $\psi$ has finite total variation, in fact $V(\psi)=\|\psi\|$, and a scalar multiple of a map with finite total variation also has finite total variation.

We say that a map $\varnothing$ between two (e.g.) $C^{*}$-algebras $\mathscr{A}$ and $\mathscr{B}$ is nuclear if $\varphi$ can be written as $\varphi=\sum \rho_{i} \otimes B_{i}$, where $\rho_{i} \in \mathscr{A}^{\prime}$ (the dual of $\mathscr{A}$ ), $B_{i} \in \mathscr{B}$, and $\sum\left\|\rho_{i}\right\|\left\|B_{i}\right\|<\infty$. The nuclear norm of $\varphi$, $\|\varphi\|$, is then defined by $\|\varphi\| \|=\inf \left\{\sum\left\|\rho_{i}\right\|\left\|B_{\imath}\right\|: \varphi=\right.$ $\left.\sum \rho_{i} \otimes B_{i}\right\}$. It was noted in Remark 1.3ii that if $\varphi$ is nuclear, then $\phi$ admits a Hahn decomposition. The proof of Proposition 1.2 shows 
that every bounded (self-adjoint) map from $\mathscr{A} \rightarrow M_{n}$ is nuclear.

Proposition 3.2. Let $\mathscr{P}: \mathscr{A} \rightarrow \mathscr{B}$ be nuclear. Then $\varphi$ has finite total variation, and $V(\varphi) \leqq \inf \left\{\sum\left\|\tau_{i}\right\|: \varphi=\sum \tau_{i} \otimes C_{i}, 0 \leqq \tau_{i} \in \mathscr{A}^{\prime}\right.$, $\left.\left\|C_{i}\right\|=1, C_{i} \in \mathscr{B}\right)$.

Proof. We can write $\varphi=\sum \rho_{i} \otimes B_{i}$, where $\sum\left\|\rho_{i}\right\|\left\|B_{i}\right\|<\infty$. By Theorem 0.2, we can, in fact, write $\varphi=\sum \tau_{i} \otimes C_{i}$, with $\sum\left\|\tau_{i}\right\|\left\|C_{i}\right\|<\infty$ and $\tau_{i} \geqq 0$. We can also assume all $\left\|C_{i}\right\|=1$. Then for $0 \leqq a \in \mathscr{A}$, we have $\varphi(a)=\sum \tau_{i}(a) C_{i}$, which implies

$$
\begin{aligned}
\varphi(a)^{*} \varphi(a) & =\sum \tau_{i, j}(a) \tau_{j}(a) C_{\imath}^{*} C_{j} \\
& =\sum \tau_{\imath}^{2}(a) C_{i}^{*} C_{i}+\sum_{i \neq j} \tau_{i}(a) \tau_{j}(a)\left[C_{j}^{*} C_{i}+C_{j}^{*} C_{i}\right] \\
& \leqq \sum \tau_{i}^{2}(a) I+\sum_{i \neq j} \tau_{i}(a) \tau_{j}(a) \cdot 2 I=\left(\sum \tau_{\imath}(a)\right)^{2} I .
\end{aligned}
$$

Hence $|\varphi(a)| \leqq\left(\sum \tau_{\imath}(a)\right) I$. So if $a_{1}, \cdots, a_{n}$ are positive, and $\sum a_{k} \leqq 1$, we see

$$
\sum_{k=1}^{n}\left|\varphi\left(a_{k}\right)\right| \leqq \sum_{k} \sum_{i} \tau_{i}\left(a_{k}\right) I=\sum_{i} \sum_{k} \tau_{i}\left(a_{k}\right) I \leqq \sum_{i} \tau_{i}(1) I=\sum_{i}\left\|\tau_{i}\right\| I .
$$

Hence $V(\varphi) \leqq \sum\left\|\tau_{i}\right\|<\infty$, so $\varphi$ has finite total variation, and we get the estimate for $V(\varphi)$.

CoROLlary. If $\varphi^{(n)}$ are as in $\S 2$, then $V\left(\varphi^{(n)}\right)=\sum_{i=1}^{n}\left\|\rho_{2}\right\|$.

Proof. The $\rho_{i}$ have disjoint support, and all $\left|A_{i}\right|=I$.

REMARK 3.3. If $\varphi$ is self-adjoint and has finite total variation, then $\varphi$ is necessarily bounded. For if not, there exist $\left\{a_{n}\right\}$ such that $\left\|a_{n}\right\| \leqq 1,\left\|\varphi\left(a_{n}\right)\right\| \rightarrow \infty$. But $\varphi$ self-adjoint implies $\varphi\left(\operatorname{Re}\left(a_{n}\right)\right)=$ $\varphi\left(\left(a_{n}+a_{n}^{*}\right) / 2\right)=\operatorname{Re}\left(\varphi\left(a_{n}\right)\right)$, and similarly $\varphi\left(\operatorname{Im}\left(a_{n}\right)\right)=\operatorname{Im}\left(\varphi\left(a_{n}\right)\right)$, so that there are self-adjoint $a_{n}$ with $\left\|a_{n}\right\| \leqq 1$ and $\left\|\varphi\left(a_{n}\right)\right\| \rightarrow \infty$. But then $a_{n}=a_{n}^{+}-a_{n}^{-}$, where $a_{n}^{+}, a_{n}^{-} \geqq 0$, and $\left\|a_{n}\right\|=\max \left(\left\|a_{n}^{+}\right\|,\left\|a_{n}^{-}\right\|\right)$. So there exist $c_{n}$ with $0 \leqq c_{n},\left\|c_{n}\right\| \leqq 1$, and $\left\|\varphi\left(c_{n}\right)\right\| \rightarrow \infty$. But $0 \leqq c_{n} \leqq 1$, so by finite total variation, $\left\|\mid \varphi\left(c_{n}\right)\right\| \| \leqq V(\varphi)<\infty$. However, $\left\|\left|\varphi\left(c_{n}\right)\right|\right\|=\left\|\varphi\left(c_{n}\right)\right\|$, since $\varphi\left(c_{n}\right)^{*}=\varphi\left(c_{n}\right)$. This contradiction completes the proof.

Proposition 3.4. Let $\left\{P_{n}\right\}$ be finite dimensional projections such that $P_{n} \uparrow I$. Let $\varphi: \mathscr{A} \rightarrow \mathscr{L}(H)$. Suppose $\varphi_{n}=P_{n} \varphi P_{n}$ has finite total variation with $V\left(\varphi_{n}\right)<M<\infty$ for all $n$. Then $\phi$ has finite total variation with $V(\varphi) \leqq M$.

Proof. For any $B \in \mathscr{L}(H), \quad\left|P_{n} B P_{n}\right| \stackrel{w}{\longrightarrow}|B|$ [2]. Hence, 
$\sum\left|P_{n} \varphi\left(a_{i}\right) P_{n}\right| \stackrel{w}{\longrightarrow} \sum\left|\varphi\left(a_{i}\right)\right|, \quad$ where $0 \leqq a_{i}, \quad \sum a_{i} \leqq 1$. So if $\left\|\sum\left|P_{n} \varphi\left(a_{i}\right) P_{n}\right|\right\| \leqq M$ for all $n$, we have $\left\|\sum\left|\varphi\left(a_{i}\right)\right|\right\| \leqq M$ also.

Note. The example $\varphi$ of $\S 2$ shows the need of a uniform constant, for $V\left(\varphi^{(n)}\right)=\sum\left\|\rho_{i}\right\| \rightarrow \infty$.

We should say that the difficulty with the notion of finite total variation occurs in attempting to show that the sum of maps with finite total variation also has finite total variation. There is, however, a tractable case.

Proposition 3.5. Let $\varphi_{1}, \varphi_{2}$ : $\mathscr{A} \rightarrow C(Y)$ be bounded. Suppose $\varphi_{1}, \varphi_{2}$ have finite total variation. Then so does $\varphi=\varphi_{1}+\varphi_{2}$.

Proof. For $f, g \in C(Y),|f+g| \leqq|f|+|g|$. Thus, $\left|\varphi_{1}(a)+\varphi_{2}(a)\right| \leqq$ $\left|\varphi_{1}(\alpha)\right|+\left|\varphi_{2}(\alpha)\right|$. The rest follows by addition.

Proposition 3.6. Let $\varphi: \mathscr{A} \rightarrow C(Y)$ be bounded. Then $\varphi$ has finite total variation.

Proof. By 3.5, we may assume $\varphi$ is self-adjoint. Let $y \in Y$, consider $\tau_{y}=\delta_{y} \circ \varphi$. Then $\tau_{y}$ is a bounded self-adjoint functional on $\mathscr{A}$, so by Theorem 0.2, $\tau_{y}=\mu_{y}^{+}-\mu_{y}^{-}$, where $\left\|\mu_{y}^{+}\right\|+\left\|\mu_{y}^{-}\right\|=$ $\left\|\tau_{y}\right\| \leqq\|\varphi\|$. Then if $a_{i} \in \mathscr{A}, 0 \leqq \alpha_{i}, \sum a_{i} \leqq 1$, we have

$$
\begin{aligned}
\sum\left|\varphi\left(a_{i}\right)\right|(y) & =\sum\left|\tau_{y}\left(a_{i}\right)\right| \leqq \sum\left(\mu_{y}^{+}\left(a_{i}\right)+\mu_{y}^{-}\left(a_{i}\right)\right) \leqq \mu_{y}^{+}(1)+\mu_{y}^{-}(1) \\
& =\left\|\mu_{y}^{+}\right\|+\left\|\mu_{y}^{-}\right\| \leqq\|\varphi\| .
\end{aligned}
$$

By taking the supremum over $y$, we obtain $\left\|\sum\left|\varphi\left(a_{i}\right)\right|\right\| \leqq\|\varphi\|$, which shows that $\varphi$ has finite total variation.

4. A Hahn decomposition theorem. We begin with a wellknown result, which we state here in the form we need (see [5]).

LEMMA 4.1. Let $X$ be a compact Hausdorff space, let $U_{1}, \cdots, U_{n}$ be an open cover of $X$. Then there are continuous functions $g_{1}, \cdots, g_{n}$ on $X$ so that $0 \leqq g_{i} \leqq 1, \sum g_{i} \equiv 1$, and support of $g_{i} \subseteq U_{i}$. Furthermore, if the cover is nonredundant, i.e., $\forall j \mathbf{U}_{i \neq j} U_{i} \neq X$, then there are $x_{1}, \cdots, x_{n} \in X$ such that $g_{i}\left(x_{j}\right)=\delta_{i j}$.

From now on, we will assume all partitions of unity are constructed with respect to nonredundant covers, and so the word "cover" will denote a nonredundant cover, with associated points $\left\{x_{i}\right\}$.

Note, therefore, that given a cover $U_{1}, \cdots, U_{n}$ and associated 
partition of unity $g_{1}, \cdots, g_{n}$, we have $\left\|\sum \alpha_{i} g_{i}\right\|=\sup \left|\alpha_{i}\right|$. For, $\left|\sum \alpha_{i} g_{i}(x)\right| \leqq \sum\left|\alpha_{i}\right| g_{i}(x) \leqq \sup \left|\alpha_{i}\right| \sum g_{i}(x)=\sup \left|\alpha_{i}\right| ;$ but if $\left|\alpha_{i_{0}}\right|=$ $\sup \left|\alpha_{i}\right|$, then by $4.1,\left|\sum \alpha_{i} g_{\imath}\left(x_{i_{0}}\right)\right|=\left|\alpha_{i_{0}}\right|$.

Lemma 4.2. Let $\left(U_{i}, g_{i}\right)$ be a cover and associated partition of unity. Then for $F \in C(X)$, the map $Q: F \rightarrow \sum F\left(x_{i}\right) g_{i}$ is a positive linear projection of norm 1 from $C(X)$ onto the span of the $\left\{g_{i}\right\}$.

Proof. Let $Q(F)=\sum F\left(x_{i}\right) g_{i}$. Then $Q\left(g_{j}\right)=g_{j}$, and if $F \geqq 0$, $Q(F) \geqq 0$. Clearly, $Q(1)=1$, and the above note shows $\|Q\| \leqq 1$ ([3].).

LEMma 4.3. Let $\varphi: C(X) \rightarrow \mathscr{B}$ have finite total variation. Then given $\left(U_{i}, g_{i}\right)$ a cover and associated partition of unity, there exists a positive linear map $\Pi: \operatorname{span}\left\{g_{i}\right\} \rightarrow \mathscr{B}$ so that $\|\Pi\| \leqq V(\varphi)$, and, if $\varphi$ is self-adjoint, $\Pi \geqq\left.\varphi\right|_{\left.\operatorname{span} \mid g_{i}\right)}$.

Proof. Let $\Pi\left(\sum \alpha_{i} g_{i}\right)=\sum \alpha_{i}\left|\varphi\left(g_{i}\right)\right|$. Clearly $\Pi$ is linear, and $\sum \alpha_{i} g_{i} \geqq 0$ iff all $\alpha_{i} \geqq 0$; it is trivial that all $\alpha_{i} \geqq 0$ implies $\sum \alpha_{i} g_{i} \geqq 0$, and conversely, we need only evaluate at the points $\left\{x_{j}\right\}$. Hence $\Pi$ is positive, and if $\phi$ is self-adjoint, $\Pi \geqq\left.\varphi\right|_{\operatorname{span}\left\{g_{i} \mid\right.}$, for if $\sum \alpha_{i} g_{i} \geqq 0,(\Pi-\varphi)\left(\sum \alpha_{i} g_{i}\right)=\sum \alpha_{i}\left(\left|\varphi\left(g_{i}\right)\right|-\varphi\left(g_{i}\right)\right) \geqq 0$, since all $\alpha_{i} \geqq 0$, and $g_{i} \geqq 0 \Longrightarrow \varphi\left(g_{i}\right)^{*}=\varphi\left(g_{i}\right)$.

Now, we can extend $\Pi$ to $\widetilde{\Pi}: C(X) \rightarrow \mathscr{B}$ by defining $\widetilde{\Pi}=\Pi \circ Q$, $Q$ as in 4.2. Then $\|\Pi\| \leqq\|\widetilde{\Pi}\|$ since $\widetilde{\Pi}$ extends $\Pi$, and $\|\widetilde{\Pi}\| \leqq\|\Pi\|$ since $\|Q\| \leqq 1$. Thus $\|\widetilde{\Pi}\|=\|\Pi\|$. But $\widetilde{\Pi}$ is positive, since $Q$ and $\Pi$ are, so by [1], $\|\widetilde{\Pi}\|=\|\widetilde{\Pi}(1)\|$. However, $1 \in \operatorname{span}\left\{g_{i}\right\}$, so $\widetilde{\Pi}(1)=\Pi(1)$, so $\|\Pi\|=\|\Pi(1)\|=\left\|\sum\left|\varphi\left(g_{i}\right)\right|\right\| \leqq V(\varphi)$.

We now proceed with the main result of this paper.

THEOREM 4.4. Let $\varphi: C(X) \rightarrow \mathscr{R}$ be a bounded self-adjoint linear map with finite total variation, where $\mathscr{R}$ is any von Neumann algebra. Then $\varphi$ admits a Hahn decomposition, $\varphi=\phi^{+}-\phi^{-}$, where $\varphi^{+}, \varphi^{-}$are bounded positive linear maps into $\mathscr{R}$, and $\left\|\varphi^{+}\right\| \leqq V(\varphi)$.

Proof. It suffices to find a positive linear map $\varphi^{+}$into $\mathscr{R}$ such that $\left\|\varphi^{+}\right\| \leqq V(\varphi)$, and $\varphi^{+} \geqq \varphi$, for then we may take $\varphi^{-}=\varphi^{+}-\varphi$.

Let $\mathscr{C}$ be the family of all nonredundant covers of $X$ (and corresponding partitions of unity), partially ordered by inclusion of the covers. For $P \in \mathscr{C}$, let $Q_{P}$ and $\Pi_{P}$ denote the mappings of Lemmas 4.2 and 4.3 , respectively. Let $\widetilde{\Pi}_{P}=\Pi_{P} \circ Q_{P}$, then the proof of 4.3 shows $\widetilde{\Pi}_{P}: C(X) \rightarrow \mathscr{R}$ is positive, $\left\|\widetilde{\Pi}_{P}\right\|=\left\|\Pi_{P}\right\| \leqq V(\varphi)$ and $\Pi_{P} \geqq\left.\varphi\right|_{P}$, so by setting $\widetilde{\varphi}_{P}=\varphi \circ Q_{P}$, we have $\left\|\widetilde{\varphi}_{P}\right\| \leqq\|\varphi\|$ and $\widetilde{\Pi}_{P} \geqq \widetilde{\varphi}_{P}$. 
We claim $Q_{P} \stackrel{s}{\rightarrow}$ id, i.e., $\forall f \in C(X), Q_{P}(f) \rightarrow f$. Since $X$ is compact, $f$ is uniformly continuous, so there is a cover $P=\left\{U_{1}, \cdots, U_{n}\right\}$ of $X$ such that $x, y \in U_{i} \Rightarrow|f(x)-f(y)| \leqq \varepsilon$, where $\varepsilon>0$ is arbitrary. Then let $x \in X$, so

$$
\begin{aligned}
\left|f(x)-Q_{P}(f)(x)\right| & =\left|f(x)-\sum f\left(x_{j}\right) g_{j}(x)\right| \\
& =\left|\sum\left(f(x)-f\left(x_{j}\right)\right) g_{j}(x)\right| \leqq \sum\left|f(x)-f\left(x_{j}\right)\right| g_{j}(x) .
\end{aligned}
$$

Fix a $j_{0}$ : if $x \in U_{j_{0}}$, then $\left|f(x)-f\left(x_{j_{0}}\right)\right|<\varepsilon$; if $x \notin U_{j_{0}}$, then $g_{j_{0}}(x)=0$. In either case, $\left|f(x)-f\left(x_{j_{0}}\right)\right| g_{j_{0}}(x) \leqq \varepsilon g_{j_{0}}(x)$. Summing, we have $\mid f(x)-Q_{P}(f)(x) \leqq \sum \varepsilon g_{j}(x)=\varepsilon$. Thus $\left\|f-Q_{P}(f)\right\| \leqq \varepsilon$, and, by refinement of the cover, this also holds for all $P^{\prime} \geqq P$, proving our claim.

In particular, since $\varphi$ is continuous, we see that $\widetilde{\varphi}_{P}(f) \rightarrow \varphi(f)$, and since any subnet of a convergent net is convergent, we see that $\widetilde{\varphi}_{P_{\alpha}}(f) \rightarrow \varphi(f)$ for any subnet $\left\{P_{\alpha}\right\} \subseteq \mathscr{C}$.

Now, for any $K>0$, the set of all positive linear maps from $C(X)$ into $\mathscr{R}$, with norm $\leqq K$, is a compact set in the BW-topology (this is just a variant of Alaoglu's theorem, see [1]). Hence, the partial ordering on $\mathscr{C}$ makes $\left\{\widetilde{\Pi}_{P}\right\}$ into a net, and by the above comment, with $K=V(\varphi)$, there is a cluster point $\widetilde{I}$ of $\left\{\widetilde{\Pi}_{P}\right\}$. Then $\widetilde{\Pi}$ is positive, $\|\widetilde{\Pi}\| \leqq V(\varphi)$, and there is a cofinal set $\left\{P_{\alpha}\right\}$ such that $\widetilde{\Pi}_{P_{\alpha}} \stackrel{B W}{\longrightarrow} \tilde{\Pi}$.

Let $\tau_{\alpha}=\widetilde{\Pi}_{P_{\alpha}}-\widetilde{\varphi}_{P_{\alpha}}$, then $\tau_{\alpha} \geqq 0$, and $\left\|\tau_{\alpha}\right\| \leqq\left\|\widetilde{\Pi}_{P_{\alpha}}\right\|+\left\|\widetilde{\varphi}_{P_{\alpha}}\right\| \leqq$ $V(\phi)+\|\varphi\|$. By the above comments, we can choose a cluster point $\tau$ of $\left\{\tau_{\alpha}\right\}$ which is positive. Since $\tau_{\alpha}=\widetilde{I}_{P_{\alpha}}-\widetilde{\varphi}_{P_{\alpha}}$, and the $\widetilde{\Pi}_{P_{\alpha}}$ converge to $\widetilde{\Pi}$, we have $0 \leqq \tau=\widetilde{\Pi}-\widetilde{\phi}$, where $\widetilde{\varphi}$ is the corresponding cluster point of the $\widetilde{\varphi}_{P_{\alpha}}$.

But we have that for this (or any) subnet, $\widetilde{\varphi}_{P_{\alpha}} \stackrel{s}{\rightarrow} \varphi$, so we have $\widetilde{\Pi}_{P_{\alpha}}(f)-\widetilde{\varphi}_{P_{\alpha}}(f) \rightarrow \widetilde{\Pi}(f)-\varphi(f)$. Thus, we see that $0 \leqq \tau=\widetilde{\Pi}-\varphi$, i.e., $\widetilde{\Pi}$ dominates $\varphi$. We may then choose $\varphi^{+}=\widetilde{\Pi}$, yielding the desired Hahn decomposition.

THEOREM 4.5. Let $\varphi: C(X) \rightarrow C(\Omega)$, where $C(\Omega)$ is a von Neumann algebra. Then the following are equivalent:

(i) $\varphi$ is bounded; (ii) $\varphi$ has finite total variation; (iii) $\varphi$ admits a Hahn decomposition; (iv) $\varphi$ is completely bounded.

Proof. Theorem 4.4, Propositions 3.5 and 3.6, and Lemma 1.5.

Note. Oscar Lanford has informed me that (ii) $\Rightarrow$ (iii) of Theorem 4.5 fails if $C(\Omega)$ is not a von Neumann algebra.

REMARKS 4.6 (i). For the mappings $\varphi^{(n)}$ of $\S 2$, it follows that 
we can write $\varphi^{(n)}=\varphi^{+}-\varphi^{-}$, where $\varphi^{+}, \varphi^{-}$are positive and $1 / 2 \sum\left\|\rho_{i}\right\| \leqq\left\|\varphi^{+}\right\| \leqq \sum\left\|\rho_{i}\right\|$.

(ii) There is another interesting way of ordering the nonredundant partitions of unity. For any $\varepsilon>0$, we say $\left(U_{i}, g_{i}\right) \leqq\left(V_{j}, h_{j}\right)$ iff (1) the $V_{j}$ refine the $U_{i}$ and (2) $x, y \in V_{j} \Rightarrow\left|g_{i}(x)-g_{i}(y)\right|<\varepsilon \forall i$. It is not difficult to show that $\stackrel{\varepsilon}{\lessgtr}$ is, in fact, a partial order. The relation $\leqq$ has the following two interesting properties:

( a ) $\left(U_{i}, g_{\imath}\right) \leqq\left(V_{j}, h_{j}\right) \Longrightarrow\left(U_{i}, g_{i}\right) \leqq\left(V_{j}, h_{j}\right) \forall \varepsilon^{\prime} \geqq \varepsilon$;

(b) if $\left(U_{i}, g_{i}\right) \leqq\left(V_{j}, h_{j}\right)$ and we write $G=\operatorname{span}\left\{g_{i}\right\}, H=\operatorname{span}\left\{h_{j}\right\}$, then $\sup _{g \in G} \inf _{h \in I I}\|g-h\|<\varepsilon$, i.e., $G$ is almost a subspace of $H$. The usual order relation on the partitions of unity does not have a property resembling property (b).

We now order $R^{+} \times \mathscr{P}$ by $\left[\varepsilon,\left(U_{i}, g_{i}\right)\right] \leqq\left[\varepsilon^{\prime},\left(V_{j}, h_{j}\right)\right]$ iff $(1) \varepsilon^{\prime} \leqq \varepsilon$ and (2) $\left(U_{\imath}, g_{\imath}\right) \leqq\left(V_{j}, h_{j}\right)$. Given a partition $\left(U_{i}, g_{\imath}\right)$ and $f \in C(X)$, $\|f\| \leqq 1$, such that $\inf _{g \in \operatorname{span}\left\{g_{i}\right\}}\|f-g\|<\varepsilon$, note that for all $\left[\varepsilon^{\prime},\left(V_{j}, h_{j}\right)\right] \geqq\left[\varepsilon,\left(U_{i}, g_{i}\right)\right]$, then $\inf _{h \in \operatorname{span}\left\{h_{\imath}\right\}}\|f-h\|<2 \varepsilon$.

We then set $S_{(\varepsilon, P)}=\frac{\|h\| \leqq 1}{\bigcup_{\left(\varepsilon^{\prime}, P^{\prime}\right) \geqq(\varepsilon, P)} \widetilde{\Pi}_{P^{\prime}}}$, where the closure is taken in the $B W$-topology. Then we take $\tilde{\Pi} \in \bigcap S_{(\varepsilon, P)}$, for each $S_{(\varepsilon, P)}$ is $B W$-compact, and the finite intersection property applies.

By passing to a subnet, we can show $\widetilde{\Pi} \geqq \widetilde{\varphi}$, again using $\tau_{P}=\Pi_{P}-\varphi_{P}$, where $\widetilde{\varphi}$ is a cluster point of $\left\{\varphi_{P}\right\}$. Then it is not difficult to show $\widetilde{\varphi}=\varphi$, i.e., $Q_{P} \stackrel{s}{\rightarrow}$ id again.

THEOREM 4.7. Let $\varphi: C(X) \rightarrow \mathscr{R}$, a von Neumann subalgebra of $\mathscr{L}(H)$, be self-adjoint, linear with finite total variation. Then $\forall f \in C(X), \varphi(f)=V^{*} \pi(f) W$, where $K$ is another Hilbert space, $W: H \rightarrow K, \pi$ is a *representation of $C(X)$ on $K$, and $V^{*}: K \rightarrow H$. In particular, $\varnothing$ is completely bounded.

Proof. If we know that $\phi=V * \pi W$, it is easy to see that $\varphi$ is completely bounded. For

$$
\left\|\varphi \otimes \mathrm{id}_{n}\right\| \leqq\left\|V^{*} \otimes I_{n}\right\|\left\|\Pi \otimes \mathrm{id}_{n}\right\|\left\|W \otimes I_{n}\right\|=\left\|V^{*}\right\|\|\Pi\|\|W\| .
$$

By Theorem 4.4, $\varphi=\varphi^{+}-\varphi^{-}$, where $\varphi^{+}, \varphi^{-}$are positive. Two fundamental theorems of Stinespring assert that every positive map of $C(X)$ is completely positive, and hence of the form $T^{*} \sigma T$, where $T: H^{\prime} \rightarrow K^{\prime}$, and $\sigma$ is a ${ }^{*}$-representation on $K^{\prime}([7])$. So $\phi^{+}=V_{1}^{*} \pi_{1} V_{1}$ $\left(\pi_{1}\right.$ on $\left.K_{1}\right)$, and $\varphi^{-}=V_{2}^{*} \pi_{2} V_{2}\left(\pi_{2}\right.$ on $\left.K_{2}\right)$. For $z \in H$ let $W(z)=$ $\left(V_{1} z,-V_{2} z\right) \in K_{1} \oplus K_{2}, \quad \pi(f)=\pi_{1}(f) \oplus \pi_{2}(f) \quad$ on $\quad K_{1} \oplus K_{2}, \quad$ and $V^{*}(\xi, \eta)=V_{1}^{*} \xi+V_{2}^{*} \eta$ for $(\xi, \eta) \in K_{1} \oplus K_{2}$. Then $\varphi=V^{*} \pi W$ as desired. 
Theorem 4.7 may be regarded as a generalization of Stinespring's theorem [7].

We plan to discuss uniqueness of the Hahn decomposition in another paper.

\section{REFERENCES}

1. William B. Arveson, Subalgebras of $C^{*}$-algebras, Acta Mathematica 123 (1969), $141-224$.

2. J. Dixmier, Les algèbres d'operateurs dans l'espace Hilbertien, Gauthiers-Villars, Paris, 1957.

3. — Les $C^{*}$-algèbres et leurs representations, Gauthiers-Villars, Paris, 1964.

4. A. Grothendieck, Un résultat sur le dual d'une $C^{*}$-algèbre, J. Math. Pures Appl., 36 (1957), 97-108.

5. John L. Kelley, General Topology, Van Nostrand, Princeton, 1955.

6. Serge Lange, Algebra, Addison-Wesley, Reading, 1967.

7. W. Forrest Stinespring, Positive functions on $C^{*}$-algebras, Proc. Amer. Math. Soc., 6 (1955), 211-216.

Received November 19, 1973.

Wayne State University 



\section{PACIFIC JOURNAL OF MATHEMATICS}

\section{EDITORS}

RICHARD ARENS (Managing Editor) University of California

Los Angeles, California 90024

R. A. Beaumont

University of Washington

Seattle, Washington 98105
J. DUGUNDJI

Department of Mathematics University of Southern Californı

Los Angeles, California 90007

D. Gilbarg and J. Milgram

Stanford University

Stanford, California 94305

\section{ASSOCIATE EDITORS}
E. F. BECKENBACH
B. H. NeumanN
F. WOLF
K. YosHIDA

\section{SUPPORTING INSTITUTIONS}

UNIVERSITY OF BRITISH COLUMBIA

CALIFORNIA INSTITUTE OF TECHNOLOGY

UNIVERSITY OF CALIFORNIA

MONTANA STATE UNIVERSITY

UNIVERSITY OF NEVADA

NEW MEXICO STATE UNIVERSITY

OREGON STATE UNIVERSITY

UNIVERSITY OF OREGON

OSAKA UNIVERSITY
UNIVERSITY OF SOUTHERN CALIFORNIA

STANFORD UNIVERSITY

UNIVERSITY OF TOKYO

UNIVERSITY OF UTAH

WASHINGTON STATE UNIVERSITY

UNIVERSITY OF WASHINGTON

* * * *

AMERICAN MATHEMATICAL SOCIETY

NAVAL WEAPONS CENTER 


\section{Pacific Journal of Mathematics \\ Vol. 65, No. $1 \quad$ September, 1976}

David Lee Armacost, Compactly cogenerated LCA groups ............. 1

Sun Man Chang, On continuous image averaging of probability measures ...... 13

J. Chidambaraswamy, Generalized Dedekind $\psi$-functions with respect to a

polynomial. II................................... 19

Freddy Delbaen, The Dunford-Pettis property for certain uniform algebras ..... 29

Robert Benjamin Feinberg, Faithful distributive modules over incidence

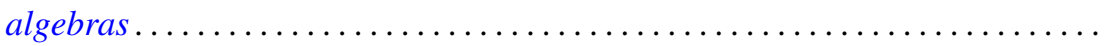

Paul Froeschl, Chained rings . . . . . . . . . . . . . . . . . . . .

John Brady Garnett and Anthony G. O'Farrell, Sobolev approximation by a sum

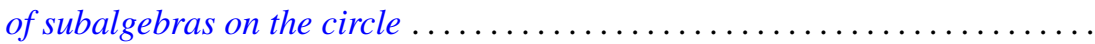

Hugh M. Hilden, José M. Montesinos and Thomas Lusk Thickstun, Closed

oriented 3-manifolds as 3-fold branched coverings of $S^{3}$ of special type.....

Atsushi Inoue, On a class of unbounded operator algebras ................

Peter Kleinschmidt, On facets with non-arbitrary shapes.

Narendrakumar Ramanlal Ladhawala, Absolute summability of Walsh-Fourier

series

Howard Wilson Lambert, Links which are unknottable by maps . . . . . . . . . . .

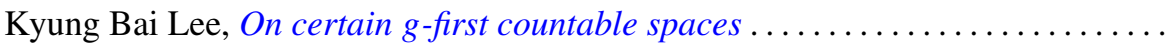

Richard Ira Loebl, A Hahn decomposition for linear maps .................

Moshe Marcus and Victor Julius Mizel, A characterization of functionals on $W_{1}^{p}$ possessing autonomous kernels. I . .

James Miller, Subordinating factor sequences and convex functions of several

variables.

Keith Pierce, Amalgamated sums of abelian l-groups ...

Jonathan Rosenberg, The $C^{*}$-algebras of some real and $p$-adic solvable

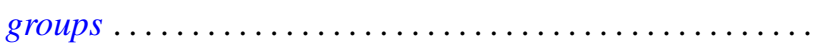

Hugo Rossi and Michele Vergne, Group representations on Hilbert spaces defined

in terms of $\partial_{b}$-cohomology on the Silov boundary of a Siegel domain . .

Mary Elizabeth Schaps, Nonsingular deformations of a determinantal

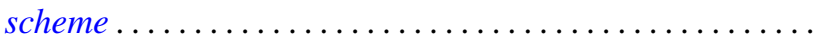

S. R. Singh, Some convergence properties of the Bubnov-Galerkin method...

Peggy Strait, Level crossing probabilities for a multi-parameter Brownian

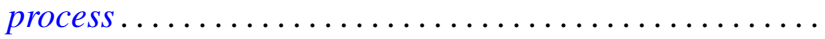

Robert M. Tardiff, Topologies for probabilistic metric spaces .

Benjamin Baxter Wells, Jr., Rearrangements of functions on the ring of integers of

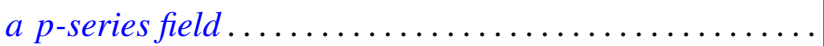

Robert Francis Wheeler, Well-behaved and totally bounded approximate identities for $C_{0}(X)$.

Delores Arletta Williams, Gauss sums and integral quadratic forms over local

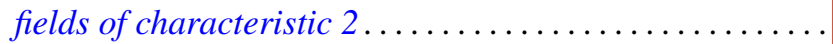

John Yuan, On the construction of one-parameter semigroups in topological 